Jun-ichi Kadokawa*

\title{
Enzymatic preparation of functional polysaccharide hydrogels by phosphorylase catalysis
}

https://doi.org/10.1515/pac-2017-0802

\begin{abstract}
This article reviews enzymatic preparation of functional polysaccharide hydrogels by means of phosphorylase-catalyzed enzymatic polymerization. A first topic of this review deals with the synthesis of amylose-grafted polymeric materials and their formation of hydrogels, composed of abundant natural polymeric main-chains, such as chitosan, cellulose, xantham gum, carboxymethyl cellulose, and poly $(\gamma$-glutamic acid). Such synthesis was achieved by combining the phosphorylase-catalyzed enzymatic polymerization forming amylose with the appropriate chemical reaction (chemoenzymatic method). An amylose-grafted chitin nanofiber hyrogel was also prepared by the chemoenzymatic approach. As a second topic, the preparation of glycogen hydrogels by the phosphorylase-catalyzed enzymatic reactions was described. When the phosphorylase-catalyzed enzymatic polymerization from glycogen as a polymeric primer was carried out, followed by standing the reaction mixture at room temperature, a hydrogel was obtained. $\mathrm{pH}$-Responsive amphoteric glycogen hydrogels were also fabricated by means of the successive phosphorylase-catalyzed enzymatic reactions.
\end{abstract}

Keywords: carbohydrates; enzyme catalysis; POLYCHAR-25; polymerization; polysaccharides.

\section{Introduction}

Polysaccharides are widely distributed on the earth and mainly act as structural materials and providers of energy [1]. Because their huge annual production, furthermore, polysaccharides are identified as very important biomass resources [2]. Accordingly, natural polysaccharides can be expected as candidates of components for the production of environmentally benign functional materials [3, 4]. For example, cellulose is the most abundant organic resource on the earth, which is composed of $\beta(1 \rightarrow 4)$-linked D-glucose (G) repeating units, has been used in a number practical applications including its use in clothing, furniture, and medical products $[5,6]$.

A variety of natural polysaccharides are known to spontaneously form hydrocolloid gels in aqueous solutions, which have characteristics close to both a liquid and to a solid [7-9] and are employed practically as stabilizers, viscous agents, and structure providers in food industries [10]. Accordingly, new gelling systems from abundant polysaccharides prepared by appropriate procedures are one of the efficient approaches to obtain environmentally benign functional materials.

Article note: A collection of invited papers based on presentations at the $25^{\text {th }}$ POLYCHAR 2017 World Forum on Advanced Materials Kuala Lumpur, Malaysia, October 9-13, 2017.

*Corresponding author: Jun-ichi Kadokawa, Graduate School of Science and Engineering, Kagoshima University, 1-21-40 Korimoto, Kagoshima 890-0065, Japan, e-mail: kadokawa@eng.kagoshima-u.ac.jp 
Amylose is a well-known abundant polysaccharide as one component of starch, which is composed of $\mathrm{G}$ repeating unit, but linked through the opposite stereoarrangement to cellulose, that is, $\alpha(1 \rightarrow 4)$ glycosidic linkage [1]. Pure amylose is hard to prepare from starch because of difficulty in complete separation from amylopectin, the other component of starch [11]. Alternatively, the enzymatic polymerization method catalyzed by phosphorylase is a powerful tool to obtain a pure amylose sample with desired molecular weight.

Phosphorylase is the enzyme that catalyzes the reversible phosphorolysis of $\alpha(1 \rightarrow 4)$-glucans at the nonreducing end, e.g. amylose, in the presence of inorganic phosphate (Pi), to yield $\alpha$-D-glucose 1-phosphate (G-1-P) (Fig. 1) [12, 13]. By means of the reversible reaction, phosphorylase is one of the enzymes which have been used practically for the polysaccharide synthesis and catalyzes enzymatic polymerization of G-1-P as a monomer using a maltooligosaccharide primer to produce $\alpha(1 \rightarrow 4)$-glucan, that is, amylose [12-18]. The polymerization is initiated from the non-reducing end of the maltooligosaccharide primer and $\mathrm{G}$ residues are consecutively transferred from the monomer to the propagating non-reducing end with inorganic phosphate (Pi) liberated by the enzymatic catalysis, according to the following reaction manner; $[\alpha(1 \rightarrow 4)-G]_{n}+\mathrm{G}-1-$ $\mathrm{P} \rightleftarrows[\alpha(1 \rightarrow 4)-G]_{n+1}+\mathrm{Pi}$. As the phosphorylase-catalyzed enzymatic polymerization belongs to the manner of chain-growth polymerization and progresses analogously to living polymerization without the occurrence of termination and chain-transfer reaction, the molecular weights of the produced amyloses can be controlled by G-1-P monomer/primer feed ratios [19].

Because the reducing end of maltooligosaccharide does not participate into the enzymatic reaction, the phosphorylase-catalyzed enzymatic polymerization can be conducted using immobilized primers, in which the reducing end is covalently linked to other substances such as polymeric chains, giving rise to amylose-grafted materials (Fig. 2) [20-22]. Furthermore, as several maltooligosaccharides are attached onto the polymeric substance, the immobilized primer produces plural amyloses by the enzymatic polymerization initiated from each non-reducing end. Glycogen, which is a high molecular weight and water soluble natural polysaccharide, also acts as a polymeric primer for the phosphorylase-catalyzed enzymatic polymerization, because it is composed of $\alpha(1 \rightarrow 4)$-glucan chains, which are interlinked by $\alpha(1 \rightarrow 6)$-glucosidic branching linkages, resulting in the presence of a number of $\alpha(1 \rightarrow 4)$-glucan non-reducing primer ends [23, 24].

Amylose acts as an energy resource in nature, but, unlike cellulose, is not required to show property as structural material. A single amylose chain exhibits water solubility, but spontaneously forms an antiparallel double helix each other owing to its helical conformation to produce a water-insoluble assembly [25, 26]. The gelling system from amylose with suitable polymeric components has been reported, and is constructed by the formation of amylose double helix as non-covalent cross-linking points through the phosphorylase-catalyzed

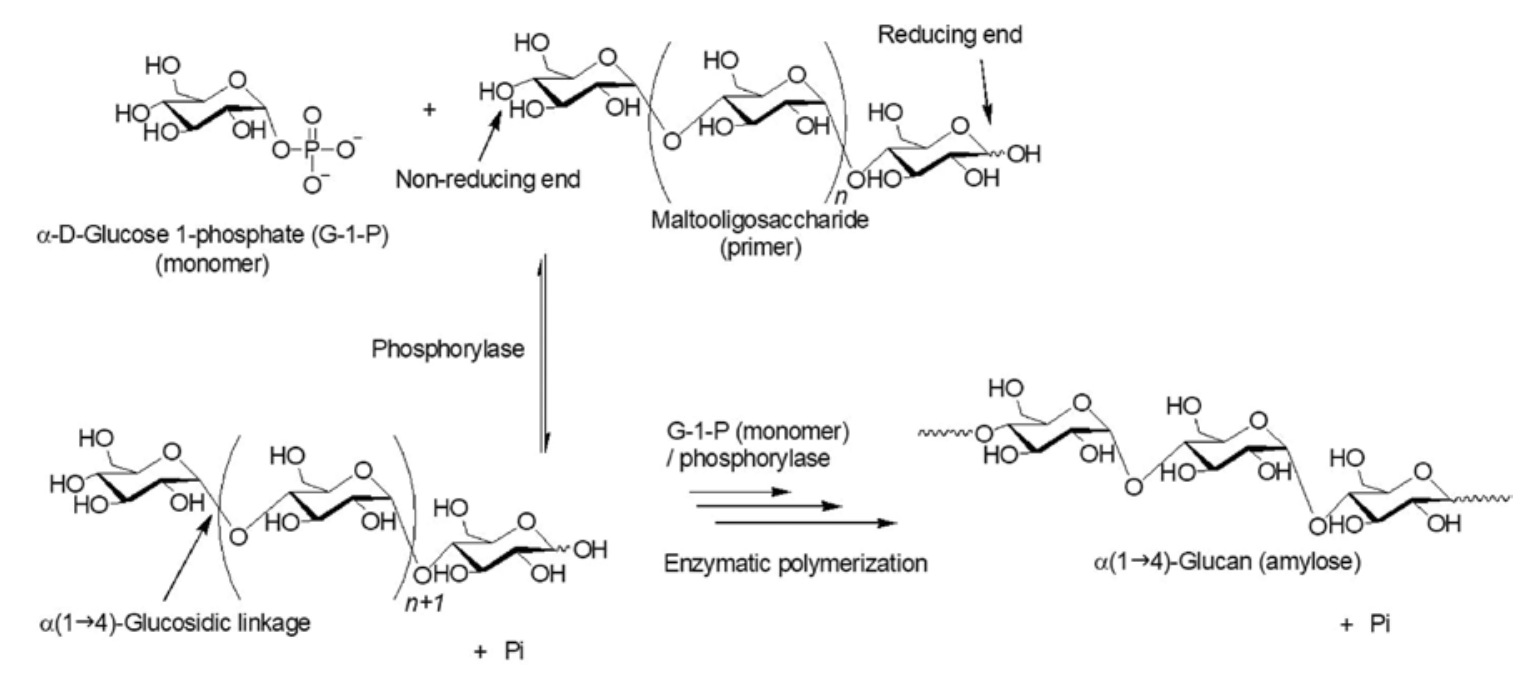

Fig. 1: Phosphorylase-catalyzed enzymatic polymerization to produce amylose. 


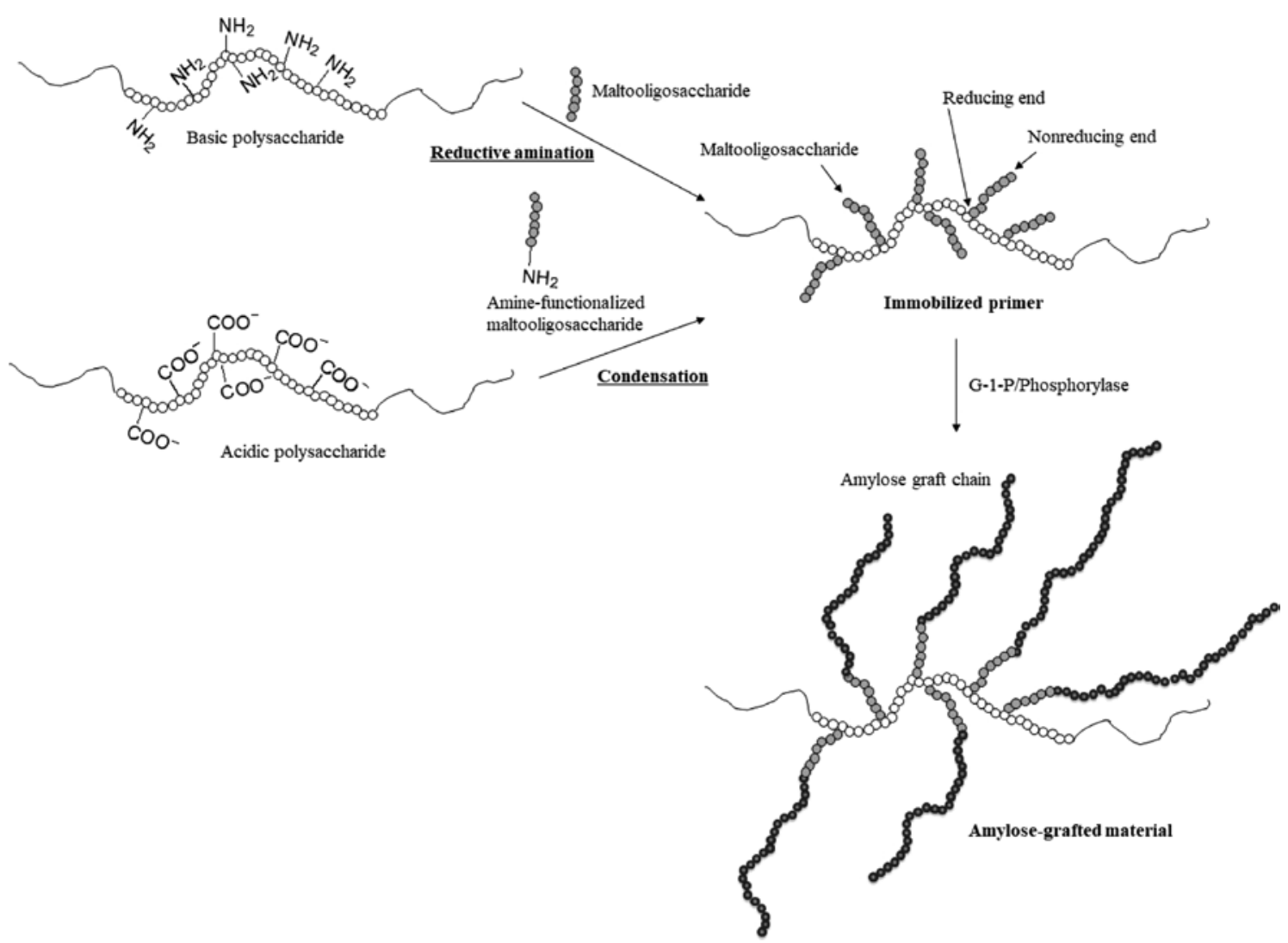

Fig. 2: Chemoenzymatic synthesis of amylose-grafted materials using immobilized primers from basic and acidic polysaccharides.

enzymatic polymerization [21, 22, 27-30]. The present review article deals with the preparation of hydrogels from amylose by means of the phosphorylase-catalyzed enzymatic polymerization.

\section{Preparation of polysaccharide hydrogels by phophorylase-catalyzed enzyamtic polymerization using immobilized primers}

Polysaccharide primers having multiple maltooligosaccharide chains have been used for the phosphorylasecatalyzed enzymatic polymerization to produce hydrogels. The maltooligosaccharide primer chains have been introduced onto the main-chain polysaccharides by the following two types of the chemical reactions; one is reductive amination of maltooligosaccharides with basic polysaccharides having amino groups using reductants and the other one is condensation of amine-functionalized maltooligosaccharides with acidic polysaccharides having carboxylate groups using condensing agents (Fig. 2) [22, 28, 31-34]. By means of the former reaction, followed by the phosphorylase-catalyzed enzymatic polymerization (chemoenzymatic approach), amylose-grafted chitosan and cellulose hydrogels have been obtained (Fig. 3) [35-37].

Chitosan is composed of $\beta(1 \rightarrow 4)$-linked D-glucosamine (GlcN) repeating units, and is prepared by $N$-deacetylation of a natural polysaccharide, chitin [38]. The reducing end of maltoheptaose $\left(G_{7}\right)$ was first reacted with amino groups at $\mathrm{C}-2$ position in chitosan by the reductive amination using $\mathrm{NaBH}_{3} \mathrm{CN}$ in a mixed solvent of aqueous acetic acid/methanol to give a maltooligosaccharide-grafted chitosan. The phosphorylase-catalyzed enzymatic polymerization of G-1-P from the maltooligosaccharide primers on the product was then carried out to obtain an amylose-grafted chitosan. When the reaction mixture was slowly dried in the vessel at $40-50^{\circ} \mathrm{C}$, a hydrogel of the product was produced. The formation of double helix cross-linking points between the amylose graft chains on the chitosan probably induced the hydrogelation. 
Basic polysaccharide main-chains

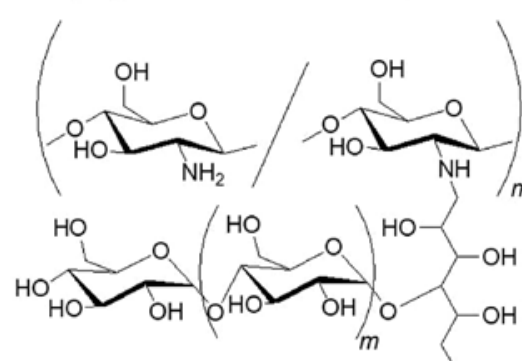

Amylose-grafted chitosan $\mathrm{OH}$

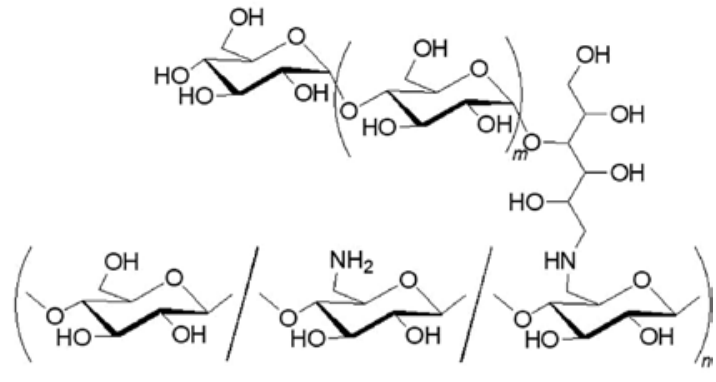

Amylose-grafted cellulose

$\underline{\text { Acidic polysaccharide main-chains }}$

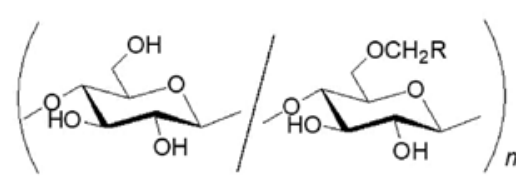

Amylose-grafted carboxymethyl cellulose
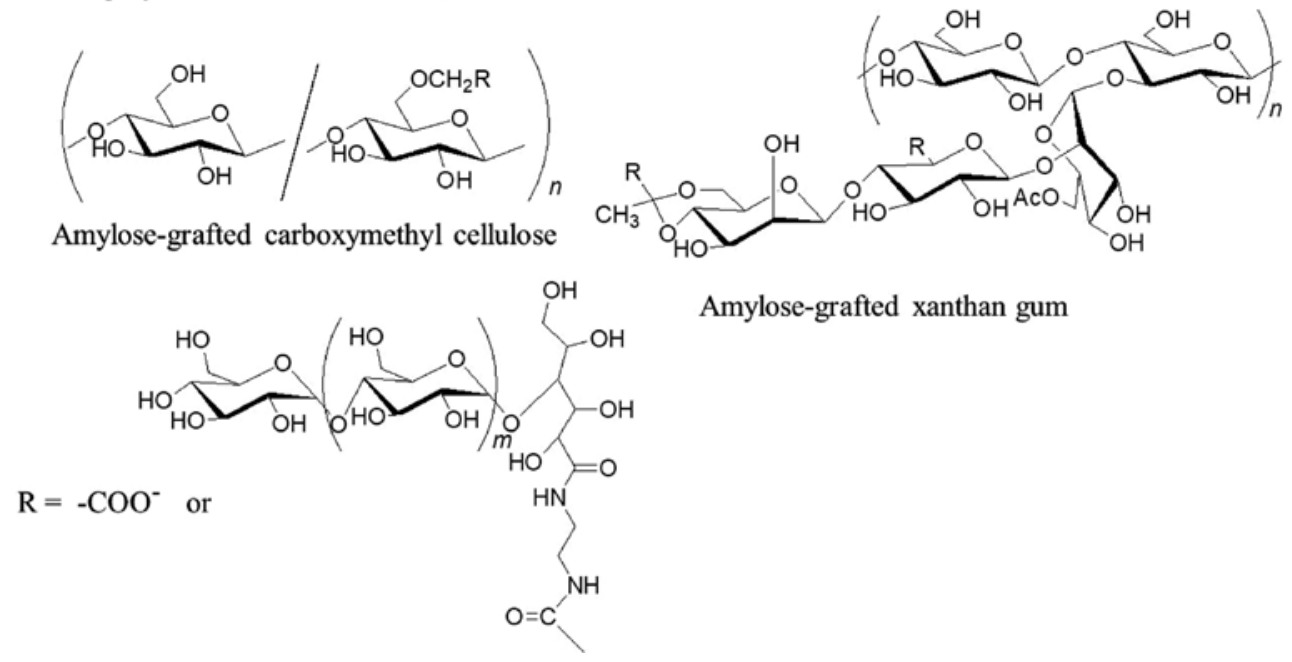

Fig. 3: Amylose-grafted heteropolysaccharides from basic and acidic polysaccharides.

The amylose-grafted cellulose hydrogel was also fabricated by the chemoenzymatic method. To conduct reductive amination for the introduction of maltooligosaccharide primers, primary hydroxy groups at C- 6 position in cellulose were first converted into amino groups by the following successive reactions; partial tosylation of hydroxy groups, displacement of the tosylates by azido groups, and reduction. Reductive amination of the produced amine-functionalized cellulose with $\mathrm{G}_{7}$ was conducted to obtain a maltooligosaccharide-grafted cellulose. The phosphorylase-catalyzed enzymatic polymerization of G-1-P from the maltooligosaccharide primers on cellulose main-chain was subsequently carried out to produce an amylose-grafted cellulose. The reaction mixture totally turned into the gel form when it was left standing on a Petri dish at room temperature for several days. The resulting hydrogel was tougher than that formed from the amylose-grafted chitosan. A solid material was obtained by drying the hydrogel under the ambient atmosphere. The addition of water to the solid resulted in the hydrogelation again. Such conversion cycle was repeated by the wetting and drying process. Moreover, the reaction mixture of the enzymatic polymerization was spread thinly on a glass plate, and subsequently left standing at room temperature, resulting in a film.

Amylose-grafted chitin nanofibers were also fabricated by the similar chemoenzymatic approach [39]. Nanofibrillated materials from native chitin, so-called chitin nanofibers, have increasingly attracted much attention and are expected to find applications as new functional materials owing to their outstanding characteristics such as lightweight, high tensile strength, and low thermal expansion coefficient [40-42]. As native chitin sources are made up from assemblies of nanostructures, several methods for disentanglement of the assemblies have efficiently been developed to obtain nanofiber dispersions in water [43-46]. It was also reported that re-dispersible amidinium chitin nanofibers were obtained from an amidinated chitin by $\mathrm{CO}_{2}$ gas bubbling with ultrasonic treatment in water [47]. For the chemoenzymatic approach, malooligosaccharide primers were introduced by reductive amination with amino groups present on the nanofibers 

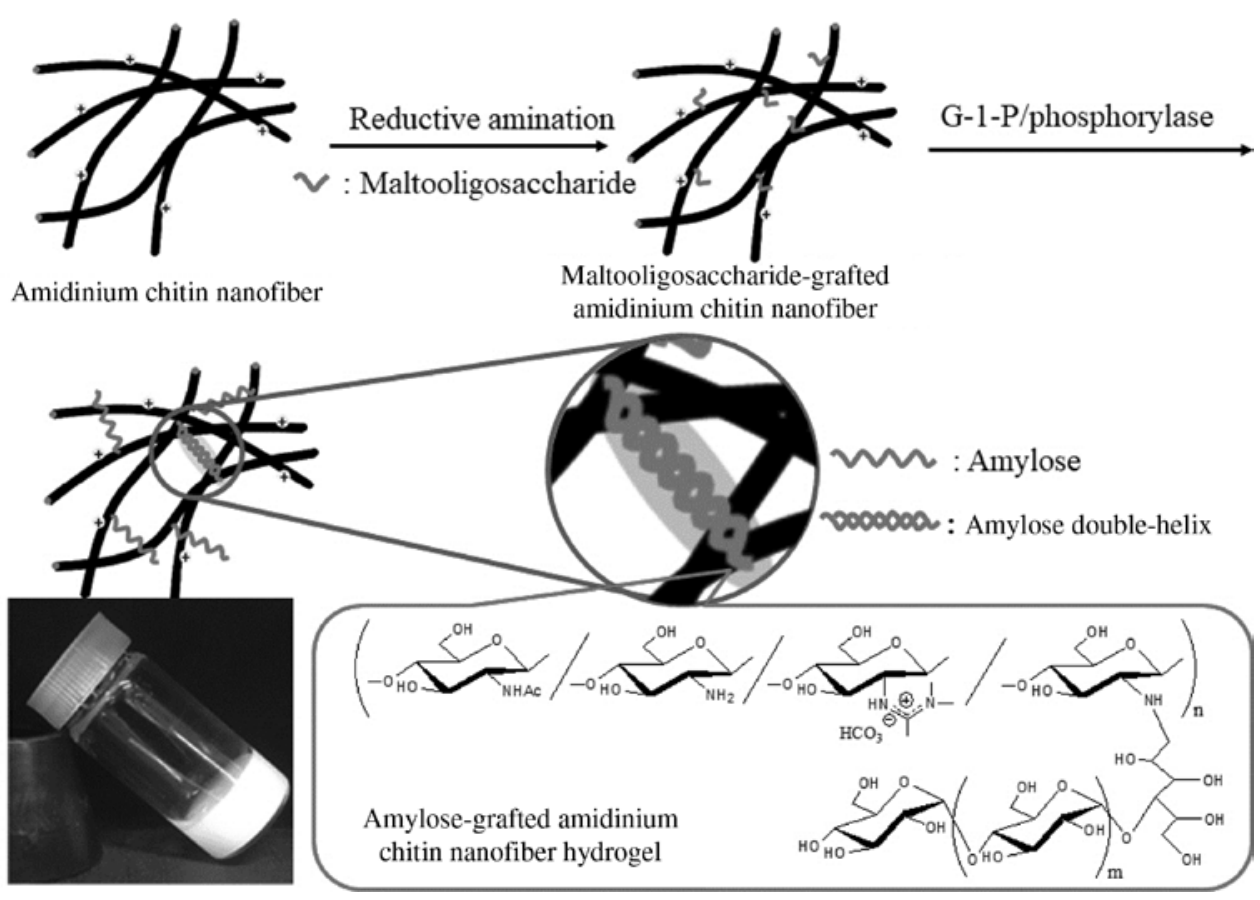

Fig. 4: Chemoenzymatic synthesis of amylose-grafted amidinium chitin nanofiber.

(Fig. 4). Grafting of amylose on the amidinium chitin nanofibers was then investigated by the phosphorylasecatalyzed enzymatic polymerization from the maltooligosaccharide graft chains to produce amylose-grafted chitin nanofiber materials. The reaction mixtures turned into hydrogels upon increasing the monomer/ primer feed ratios. The formation of double helixes from a part of amylose graft chains closely preset among the nanofibers contributed to forming the smaller networks, resulting in hydrogelation. Lyophilization of the hydrogels gave rise to the controlled microstructures, which were changed from fibrous to porous morphologies depending on the molecular weights of the amylose graft chains. Most of the amylose graft chains with the higher molecular weights, which did not participate into double helixes, formed amorphous membranes in the nanofiber networks by lyophilization, to construct the porous structure.

For the latter condensation reaction with acidic polysaccharides having carboxylate groups in the chemoenzymatic approach, functionalization of the reducing end of $\mathrm{G}_{7}$ by an amino group was necessary because it can potentially react with the carboxylates by condensation to obtain maltooligosaccharide-grafted polysaccharides (Fig. 2). First, $\mathrm{G}_{7}$ lactone was synthesized by successive $\mathrm{I}_{2} / \mathrm{KOH}$ oxidation at the reducing end, cation exchange, and intramolecular dehydrative condensation [48]. The product was then reacted with 2-azidoethylamine, followed by reduction by $\mathrm{NaBH}_{4}$, to give an amine-functionalized maltooligosaccharide at the reducing end. This substrate was reacted with xanthan gum and carboxymethyl cellulose sodium salt (NaCMC) by condensation. Then, the products were used for the phosphorylase-catalyzed enzymatic polymerization to obtain the corresponding hydrogels (Fig. 3) [49-51].

Xanthan gum, which is a water soluble polysaccharide produced by Xanthomonas campestris, has a cellulose-type main-chain $(\beta(1 \rightarrow 4)$-glucan) with trisaccharide side-chains (mannose- $\beta(1 \rightarrow 4)$-glucuronic acid- $\beta(1 \rightarrow 2)$-mannose- $\alpha(1 \rightarrow 3)$-) attached to alternating glucose units in the main-chain [52]. C-6-Position in $\alpha$-mannoside unit is acetylated and C- 4 and -6 positions in $\beta$-mannoside unit are partially pyruvated. For the chemoenzymatic approach, condensation of an amine-functionalized maltooligosaccharide with carboxylates of xanthan gum was first conducted using water-soluble carbodiimide (WSC)/ $N$-hydroxysuccinimide (NHS) as the condensing agent to produce a maltooligosaccharide-grafted xanthan gum. The phosphorylasecatalyzed enzymatic polymerization of G-1-P from the maltooligosaccharide primers on xanthan gum mainchain was then performed to obtain an amylose-grafted xanthan gum. An ion gel was formed by mixing and stirring the product with an ionic liquid, 1-butyl-3-methylimidazolium chloride, until the mixture became 
homogeneous. Exchange of dispersion media was conducted by immersing the ion gel in water to produce a hydrogel. Ionically cross-linking of carboxylates with trivalent metal cations in the hydrogel was additionally attempted by soaking it in aqueous $\mathrm{FeCl}_{3}$. When the mechanical properties of the ionically cross-linked hydrogels with $\mathrm{Fe}^{3+}$ were evaluated by compressive testing, the fracture strain values increased with increasing functionalities and DPs of the amylose graft-chains. The formation of double helixes between the amylose graft chains probably contributed to the formation of looser network structures in the hydrogels, leading to the enhancement of elasticity.

An amylose-grafted NaCMC was also synthesized by the same chemoenzymatic approach to the amylosegrafted xanthan gum. NaCMC is one of the most widely used cellulose derivatives in practical application fields such as food industry [10]. A maltooligosaccharide-grafted NaCMC was first synthesized by condensation of an amine-functionalized maltooligosaccharide with carboxylates of NaCMC in the presence of WSC/NHS. The phosphorylase-catalyzed enzymatic polymerization of G-1-P using the maltooligosaccharidegrafted NaCMC was performed in sodium acetate buffer to produce a hydrogel. Lyophilization of the hydrogel was conducted to isolate the amylose-grafted NaCMC. A film was formed by drying a thinly spread alkaline solution of the isolated product in aqueous $\mathrm{NaOH}$. The SEM image of the film showed the morphology of<smiles>CC(C)(C)NC(CCC(=O)C(C)(C)C)C(=O)O</smiles>

Poly $(\gamma$-glutamic acid $)$ (PGA)

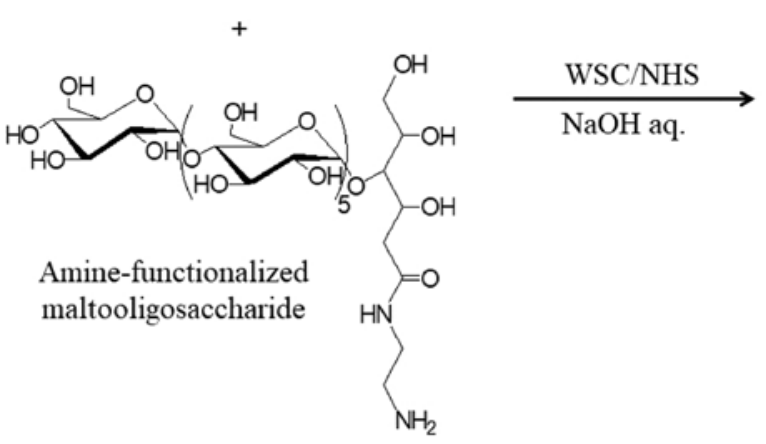

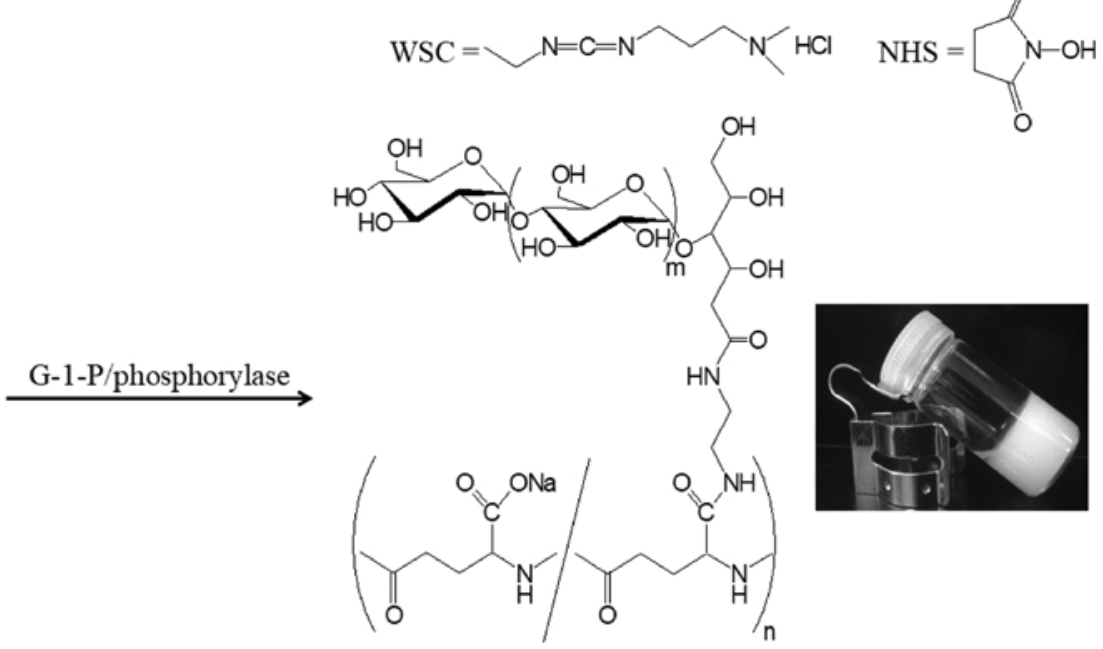

Amylose-grafted PGA

Fig. 5: Chemoenzymatic synthesis of amylose-grafted PGA. 
highly entangled nanofibers. $\mathrm{NaOH}$ present in the film was then removed by immersing in water. The SEM image of the resulting film showed that nanofibers were merged each other at interfacial area with remaining the fiber arrangements. The following self-assembling generative (bottom-up) process has been proposed for the formation of the nanofiber film. The introduction of the amylose graft chains on NaCMC prevented the construction of random-coil conformation and provided the rigid nature of the NaCMC chain. Some of these rigid materials regularly assembled in nano-scale with the slight double helix formation from the amylose graft chains on NaCMCs by drying the aqueous alkaline solution, but, the produced nano-assemblies do not aggregate further because of the prevention of the double helix formation from most of the amylose chains under the strong basic conditions. By washing out $\mathrm{NaOH}$ from the film, double helixes from amylose graft chains on the nanofibers are largely formed, resulting in merging on the surface of the fibers.

The above chemoenzymatic approach including condensation was employed for the synthesis of amylosegrafted polypeptide [53]. As the polypeptide main-chain, poly( $\gamma$-glutamic acid) (PGA) was selected because it has carboxylate groups and has been used for multifarious potential applications in foods, pharmaceuticals, healthcare, water treatment, and other fields (Fig. 5) [54, 55]. Maltooligosaccharide primers were first introduced on PGA main-chain by the condensation reaction using the WSC/NHS condensing agent in aqueous $\mathrm{NaOH}$. The phosphorylase-catalyzed enzymatic polymerization was then performed from the primer chain ends of the product to obtain amylose-grafted PGAs, which formed hydrogels in reaction media depending on monomer/primer feed ratios. The amylose graft chains formed double helixes, which acted as cross-linking points for self-assembling hydrogelation. The SEM images of the cryogels, which were prepared by lyophilization of the hydrogels showed regularly controlled porous morphologies. Furthermore, pore sizes increased with increasing monomer/primer feed ratios, while the degrees of substitution of primer on the PGA main chain did not obviously affect pore sizes.

\section{Preparation of polysaccharide hydrogels by phophorylase-catalyzed enzyamtic polymerization using natural polymeric primer, glycogen}

When the phosphorylase-catalyzed enzymatic polymerization of G-1-P from glycogen as a polymeric primer was carried out, followed by standing the reaction mixture at room temperature for $24 \mathrm{~h}$, a hydrogel was formed (Fig. 6) [56]. During the enzymatic polymerization, double helixes were formed from the elongated amylose chains among glycogen molecules, which acted as cross-linking points to give the hydrogel. The mechanical properties of the hydrogels produced by the different G-1-P/glycogen ratios were evaluated by compressive testing. The resulting stress-strain curves indicated that the properties were changed from elastic to hard and brittle with increasing the amounts of glycogen molecules. Because the number of elongated amylose chains increased with increasing amounts of glycogen, the hydrogel strength increased as more double helix cross-linking points were formed.

Lyophilziation of the hydrogels readily gave cryogels with porous morphology. Compressive testing of the cryogels showed that the mechanical properties were strengthened with increasing the amounts of glycogen. This is because of the formation of smaller networks owing to the higher cross-linking densities from the larger amounts of glycogen. A transparent film was produced by casting the alkaline solution of the cryogel on a glass plate and drying.

pH-Responsive amphoteric glycogen hydrogels were also fabricated by means of the similar approach through the phosphorylase-catalyzed enzymatic polymerization [57]. Based on the fact that phosphorylase catalyzes the enzymatic reactions using analog substrates of G-1-P such as $\alpha$-D-glucuronic acid 1-phosphate (acidic substrate) and $\alpha$-D-glucosamine 1-phosphate (basic substrate) [58, 59], amphoteric glycogens having both glucuronic acid and glucosamine units at the non-reducing ends have been synthesized by phosphorylase-catalyzed successive enzymatic reactions using the above analog substrates (Fig. 6). The functionalities of the acidic and basic units were controlled by the feed ratios. Furthermore, the products were found to exhibit the inherent isoelectric point (pI) values, which were reasonably changed in accordance with the acidic/basic unit ratios in the amphoteric products. 


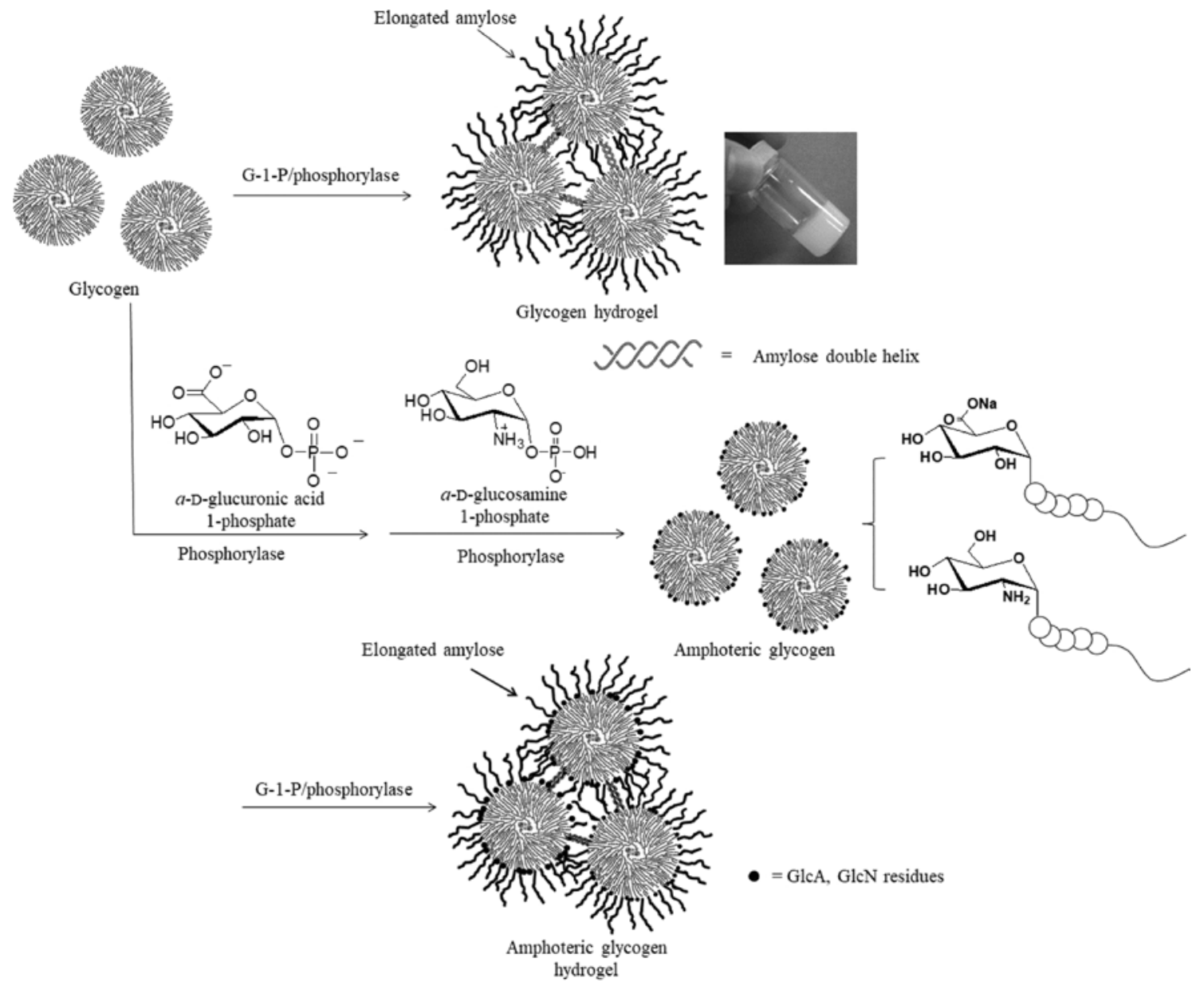

Fig. 6: Preparation of (amphoteric) glycogen hydrogels by phosphorylase-catalyzed enzymatic reactions.

Accordingly, the amphoteric glycogen hydrogels were produced by the formation of double helix crosslinking points of the elongated amylose chains by the phosphorylase-catalyzed enzymatic polymerization of G-1-P from the nonfunctionalized, non-reducing ends in the amphoteric glycogens (Fig. 6). The amphoteric hydrogels produced exhibited $\mathrm{pH}$ responsive behavior. Under strong basic conditions by addition of $\mathrm{NaOH}$ aqueous solution, the gels were solubilized by the dissociation of double helix cross-linking points. By changing $\mathrm{pH}$ to weak basic by addition of acetic acid aqueous solution, reconstruction of double helix cross-linking points occurred, resulting in re-hydrogelation. The hydrogels exhibited pH-responsive shrinking/swelling behavior. The hydrogels are shrunk at $\mathrm{pH}=\mathrm{pI}$ owing to the absence of intermolecular repulsive forces by neutral charges on the surfaces. At both weakly acidic and basic pHs, on the other hand, the surfaces of glycogens were charged, leading to swelling of the hydrogels owing to cationic/cationic or anionic/ anionic electrostatic repulsion.

\section{Conclusion}

This review article overviewed the chemoenzymatic synthesis of functional polysaccharide (amylosic) hydrogels by means of the phosphorylase-catalyzed enzymatic polymerization of G-1-P initiated from maltooligosaccharide-grafted polymeric primers. The amylose-grafted polymeric hydrogels composed of various abundant natural polymeric main-chains were synthesized by the chemoenzymatic approach. The method included the introduction of the malooligosaccharide primers on the main-chain polymers by the appropriate chemi- 
cal reactions and the following phosphorylase-catalyzed enzymatic polymerization from the primer chain ends on the products. The chemoenzymatic approach also provided the amylose-grafted chitin nanofiber hydrogel. Glycogen was used as the polymeric primer for the phosphorylase-catalyzed enzymatic polymerization, leading to hydrogelation. The amphotertic glycogen was synthesized by the successive phosphorylasecatalyzed enzymatic reactions using analog substrates, which was converted into $\mathrm{pH}$-responsive amphoteric glycogen hydrogel by the phosphorylase-catalyzed enzymatic polymerization. The amylosic hydrogels produced by the phosphorylase-catalyzed enzymatic polymerization have potentials for the practical applications in the biomedical and environmentally benign research fields because of biodegradable, eco-friendly, and non-toxic properties of amylose.

Acknowledgment: The author is indebted to the co-workers, whose names are found in references from their papers, for their enthusiastic collaborations.

\section{References}

[1] C. Schuerch. in Encyclopedia of Polymer Science and Engineering, H. F. Mark, N. Bilkales, C. G. Overberger (Eds.), pp. 87-162. John Wiley \& Sons, New York (1986).

[2] R. C. Brown, T. R. Brown. Biorenewable Resources: Engineering New Products from Agriculture. Wiley Blackwell, Chichester, West Sussex, UK (2014).

[3] A. K. Mohanty, M. Misra, L. T. Drzal. J. Polym. Environ. 10, 19 (2002).

[4] A. Rouilly, L. Rigal. J. Macromol. Sci. Polym. Rev. C42, 441 (2002).

[5] D. Klemm, B. Heublein, H. P. Fink, A. Bohn. Angew. Chem. Int. Ed. 44, 3358 (2005).

[6] A. Lejeune, T. Deprez. in Cellulose: Structure and Properties, Derivatives and Industrial Uses. Nova Science Publishers, Hauppauge, NY (2009).

[7] L. Piculell. Curr. Opin. Colloid Interface Sci. 3, 643 (1998).

[8] K. Nishinari, H. Zhang, S. Ikeda. Curr. Opin. Colloid Interface Sci. 5, 195 (2000).

[9] K. Nishinari, R. Takahashi. Curr. Opin. Colloid Interface Sci. 8, 396 (2003).

[10] A. M. Stephen, G. O. Phillips, P. A. Williams. Food Polysaccharides and their Applications. CRC/Taylor \& Francis, Boca Raton, FL (2006).

[11] R. W. Lenz. Adv. Polym. Sci. 107, 1 (1993).

[12] M. Kitaoka, K. Hayashi. Trends Glycosci. Glycotechnol. 14, 35 (2002).

[13] H. Nakai, M. Kitaoka, B. Svensson, K. Ohtsubo. Curr. Opin. Chem. Biol. 17, 301 (2013).

[14] G. Ziegast, B. Pfannemuller. Carbohydr. Res. 160, 185 (1987).

[15] K. Ohdan, K. Fujii, M. Yanase, T. Takaha, T. Kuriki. Biocatal. Biotransform. 24, 77 (2006).

[16] V. Puchart. Biotechnol. Adv. 33, 261 (2015).

[17] J. Kadokawa. Polymers 8, 138 (2016).

[18] M. Yanase, T. Takaha, T. Kuriki. J. Sci. Food Agric. 86, 1631 (2006).

[19] S. Kitamura. in The Polymeric Materials Encyclopedia, Synthesis, Properties and Applications, C. Salamone (Ed.), pp. 7915-7922. CRC Press, New York (1996).

[20] S. Kitamura, H. Yunokawa, S. Mitsuie, T. Kuge. Polym. J. 14, 93 (1982).

[21] H. Izawa, J. Kadokawa. in Interfacial Researches in Fundamental and Material Sciences of Oligo- and Polysaccharides, J. Kadoakwa (Ed.), pp. 69-86. Transworld Research Network, Trivandrum (2009).

[22] J. Kadokawa. Pure Appl. Chem. 86, 701 (2014).

[23] P. C. Calder. Int. J. Biochem. 23, 1335 (1991).

[24] D. J. Manners. Carbohydr. Polym. 16, 37 (1991).

[25] F. Eisenhaber, W. Schulz. Biopolymers 32, 1643 (1992).

[26] W. Hinrichs, G. Buttner, M. Steifa, C. Betzel, V. Zabel, B. Pfannemuller, W. Saenger. Science 238, 205 (1987).

[27] J. Kadokawa, S. Kobayashi. Curr. Opin. Chem. Biol. 14, 145 (2010).

[28] J. Kadokawa. Chem. Rev. 111, 4308 (2011).

[29] J. Kadokawa, Y. Kaneko. Engineering of polysaccharide materials - by phosphorylase-catalyzed enzymatic chain-elongation. Pan Stanford Publishing Pte Ltd., Singapore (2013).

[30] S. Shoda, H. Uyama, J. Kadokawa, S. Kimura, S. Kobayashi. Chem. Rev. 116, 2307 (2016).

[31] Y. Kaneko, J. Kadokawa. in Handbook of Carbohydrate Polymers: Development, Properties and Applications, R. Ito, Y. Matsuo (Eds.), pp. 671-691. Nova Science Publishers, Inc., Hauppauge, NY (2009).

[32] J. Kadoakwa. in Biobased Monomers, Polymers, and Materials, P. B. Smith, R. A. Gross (Eds.), pp. 237-255. ACS Symposium Series 1105; American Chemical Society, Washington, DC (2012). 
[33] J. Kadoakwa. in Green Polymer Chemistry: Biocatalysis and Materials II, H. N. Cheng, R. A. Gross, P. B. Smith (Eds.), pp. 141-161. ACS Symposium Series 1144; American Chemical Society, Washington, DC (2013).

[34] J. Kadokawa. in Cellulose and Cellulose Derivatives, M. I. H. Mondal (Ed.), pp. 299-311. Nova Science Publishers, Inc., Hauppauge, NY (2015).

[35] Y. Kaneko, S. Matsuda, J. Kadokawa. Biomacromolecules 8, 3959 (2007).

[36] S. Matsuda, Y. Kaneko, J. Kadokawa. Macromol. Rapid Commun. 28, 863 (2007).

[37] Y. Omagari, S. Matsuda, Y. Kaneko, J. Kadokawa. Macromol. Biosci. 9, 450 (2009).

[38] C. K. S. Pillai, W. Paul, C. P. Sharma. Prog. Polym. Sci. 34, 641 (2009).

[39] N. Egashira, K. Yamamoto, J.-i. Kadokawa. Polym. Chem. (2017).

[40] S. Ifuku. Kobunshi Ronbunshu 69, 460 (2012).

[41] S. Ifuku. Molecules 19, 18367 (2014).

[42] R. A. A. Muzzarelli, M. El Mehtedi, M. Mattioli-Belmonte. Marine Drugs 12, 5468 (2014).

[43] Y. Fan, T. Saito, A. Isogai. Biomacromolecules 9, 192 (2008).

[44] Y. M. Fan, T. Saito, A. Isogai. Carbohydr. Polym. 77, 832 (2009).

[45] S. Ifuku, M. Nogi, K. Abe, M. Yoshioka, M. Morimoto, H. Saimoto, H. Yano. Biomacromolecules 10, 1584 (2009).

[46] S. Ifuku, M. Nogi, M. Yoshioka, M. Morimoto, H. Yano, H. Saimoto. Carbohydr. Polym. 81, 134 (2010).

[47] K. Tanaka, K. Yamamoto, J. Kadokawa. Carbohydr. Res. 398, 25 (2014).

[48] K. Kobayashi, S. Kamiya, N. Enomoto. Macromolecules 29, 8670 (1996).

[49] T. Arimura, Y. Omagari, K. Yamamoto, J. Kadokawa. Int. J. Biol. Macromol. 49, 498 (2011).

[50] J. Kadokawa. Polymers 4, 116 (2012).

[51] D. Hatanaka, Y. Takemoto, K. Yamamoto, J.-i. Kadokawa. Fibers 2, 34 (2014).

[52] L. D. Melton, L. Mindt, D. A. Rees. Carbohydr. Res. 46, 245 (1976).

[53] T. Shouji, K. Yamamoto, J.-i. Kadokawa. Int. J. Biol. Macromol. 97, 99 (2017).

[54] I. Bajaj, R. Singhal. Bioresour. Technol. 102, 5551 (2011).

[55] A. Ogunleye, A. Bhat, V. U. Irorere, D. Hill, C. Williams, I. Radecka. Microbiology 161, 1 (2015).

[56] H. Izawa, M. Nawaji, Y. Kaneko, J. Kadokawa. Macromol. Biosci. 9, 1098 (2009).

[57] Y. Takata, K. Yamamoto, J. Kadokawa. Macromol. Chem. Phys. 216, 1415 (2015).

[58] M. Nawaji, H. Izawa, Y. Kaneko, J. Kadokawa. Carbohydr. Res. 343, 2692 (2008).

[59] Y. Umegatani, H. Izawa, M. Nawaji, K. Yamamoto, A. Kubo, M. Yanase, T. Takaha, J. Kadokawa. Carbohydr. Res. 350, 81 (2012). 